\section{Effect of passive immunization by anti-gingipain IgY on periodontal health of dogs}

\author{
Rahman A.K.M. Shofiqur, 1 \\ El-Sayed M. Ibrahim,2 Rie Isoda,1 \\ Kouji Umeda,1 Van Sa Nguyen, 1 \\ Yoshikatsu Kodama1
}

1Immunology Research Institute in Gifu, EW Nutrition Japan K.K., Gifu, Japan; 2Department of Animal Medicine, Faculty of Veterinary Medicine, Benha University, Moshtohor, Qalioubeya, Egypt

\section{Abstract}

Anti-gingipain IgY (IgY-GP), known as hyperimmune $\gamma$-livetin from egg yolk, inhibits the enzyme activity, growth and adherence of Porphyromonas gingivalis to gingival epithelial cells. Our objective was to evaluate the efficacy of IgY-GP on periodontal health of dogs. IgYGP was prepared from the egg yolk of hens immunized with the gingipain from Porphyromonas gingivalis ATCC 33277 . Two in vivo trial models were conducted on 15 adult dogs with periodontitis by giving IgY-GP-supplemented $\operatorname{dog}$ feed for 8 weeks and direct application of the IgY in dental ointment to the periodontal pockets at weekly interval for 4 weeks. Clinical parameters including gingivitis, periodontitis, oral health index, bleeding on probe (BOP), pocket depth (PD), and dental calculus removal pattern for selected premolar teeth were recorded at baseline, 4 and 8 weeks post treatment.IgY-GP showed strong crossreactivity with gingipain from Porphyromonas gulae and inhibited the enzyme activity in vitro. In the dog trials, IgY-GP resulted in significant improvement of oral health parameters including gingivitis and periodontitis scores, B0P, dental calculus removal. No adverse events during and after antibody applications were noted. Oral immunotherapy by using IgY-GP is a new promising alternative to conventional preventive and therapeutic methods to improve oral health status in dogs.

\section{Introduction}

Periodontitis is probably the single most common infectious disease in veterinary medicine especially in small animal practice. ${ }^{1}$ The disease is caused by a group of black-pigmented anaerobic bacteria. Among them, Porphyromonas gingivalis has been considered to be a major periodontal pathogen because the bacterium is more frequently detected in active lesions of periodontitis in humans ${ }^{2}$ and its subgingival implantation in mice, ${ }^{3}$ rats $^{4}$ and non-human primates ${ }^{5}$ is associated with initiation and progression of the disease.

Virulence of $P$. gingivalis is associated with the proteolytic enzymes gingipains ${ }^{6}$ that are produced as secreted or membrane-associated forms by the bacterium. ${ }^{7}$ Gingipains are cysteine proteinases that can degrade key components of the immune system. ${ }^{8}$ In addition, gingipains are important for the bacterium to proliferate and survive in the periodontal pockets. ${ }^{9}$ These facts suggest that gingipains are the most promising target for vaccination against periodontitis and related systemic diseases.

Immunotherapy by specific chicken antibodies (IgY) has been used with mixed successes against infectious diseases of viral, bacterial and fungal origin on both humans and animals. ${ }^{10-12}$ Peroral administration with IgY is an attractive approach because IgY does not activate mammalian complement or interact with mammalian Fc receptors that could mediate inflammatory response in the gastrointestinal tract. ${ }^{13}$ In recent papers we have reported that anti-gingipain IgY had preventive effect against periodontitis in human patients. ${ }^{14}$ In the present study we examined if the same IgY has effect against periodontal diseases in companion animals when used in different applications.

\section{Materials and Methods}

\section{Bacterial strains and culture conditions}

Porphyromonas gingivalis ATCC 33277, Porphyromonas gulae ATCC 51700, Porphyromonas salivosa ATCC 49407, Porphyromonas circumdentaria ATCC 51356 were obtained from the American Type Culture Collection. Porphyromonas gingivalis 381 and Porphyromonas endodontalis F2 and F5, 2 clinical isolates from the subgingival plaque of adult dog, ${ }^{15}$ were kindly provided by University of Hokkaido (Department of Disease Control and Molecular Epidemiology, Health Sciences University of Hokkaido, Japan). All strains were maintained anaerobically on Brucella HK agar (Kyokuto Pharmaceutical, Tokyo, Japan) supplemented with $10 \%$ horse blood.

\section{Preparation of gingipain and anti- gingipain antibody}

P. gingivalis ATCC 33277 was used for production of gingipain (GP) and IgY-GP according to the methods described previously.16 Partially purified IgY-GP is a polyclonal preparation and control IgY (prepared from nonimmunized chicken eggs) samples were pre-
Correspondence: Dr. A.K.M. Shofiqur Rahman, Immunology Research Institute in Gifu, EW Nutrition Japan K.K., 839-7, Sano, Gifu city 5011101, Japan.

Tel. +81.58.2357303 - Fax: +81.58.2357505.

E-mail: rahman@ew-nutrition.co.jp

Key words: Gingipain, IgY, Periodontitis, Pet, Porphyromonas gingivalis.

Acknowledgements: this study was funded by Immunology Research Institute in Gifu. The authors would like to thank the Kyodoken Institute for supporting the in vivo dog model study in their animal center; they also would like to thank Dr. Emiko Isogai, Health Sciences University of Hokkaido for providing with Prophyromonas endodontalis F2 and F5 isolates and Showa Yakuhin Kako Co., Ltd., Japan, for supplying the dental ointment.

Conflict of interest: the authors report no conflicts of interest.

Received for publication: 8 December 2010. Accepted for publication: 8 September 2011.

This work is licensed under a Creative Commons Attribution 3.0 License (by-nc 3.0).

(C) Copyright R.A.K.M. Shofiqur et al., 2010

Licensee PAGEPress, Italy

Veterinary Science Development 2011; 1:e8 doi:10.4081/vsd.2011.e8

pared from egg yolk by chloroform extraction and ammonium sulfate precipitation. 17 The antigen and antibody protein concentration was determined by the Bio-Rad protein assay system (Bio-Rad laboratories, Berkeley, CA, USA). Enzyme linked immunosorbent assay (ELISA) was used to check the titer of the specific antibody IgY-GP as described previously. 16

\section{In vitro assays for IgY-GP}

An indirect ELISA was used to determine the activity of IgY-GP against $P$. gingivalis, $P$. gulae, $P$. salivosa, $P$. circumdentaria and $P$. endodontalis. The bacteria were grown in enriched trypticase soy broth medium, harvested by centrifugation, washed and disrupted by sonication as described previously. 16 The obtained preparations were adjusted to $5 \mu \mathrm{g} / \mathrm{mL}$ concentration with $0.05 \mathrm{M}$ carbonate buffer $(\mathrm{pH} 9.6)$ and used to coat ELISA plates (100 $\mu \mathrm{L} /$ well) at $4^{\circ} \mathrm{C}$ for $18 \mathrm{~h}$. The indirect ELISA was performed as described previously. A similar procedure was used with purified gingipains from $P$. gingivalis as coating antigen. The highest dilution of IgY solutions showing an OD of more than 0.2 was used as the cut-off value for a positive reaction.

To examine the enzyme inhibition effect of IgY samples the following procedure was used. Gingipain preparations from various Porphy- 
romonas spp. were activated in a buffer consisting of $200 \mathrm{mM}$ HEPES, $(\mathrm{pH} 7.6), 5 \mathrm{mM}$ $\mathrm{CaCl}_{2}$ and $10 \mathrm{mM}$ cysteine for $5 \mathrm{~min}$ at $37^{\circ} \mathrm{C}$, mixed with IgY-GP or control IgY $(50 \mathrm{mg} / \mathrm{mL})$ in the same buffer, and incubated at $4^{\circ} \mathrm{C}$ for 1 h. The substrate $\mathrm{N}-\alpha$-Benzoyl-L-arginine- $p$ nitroanilide (BApNA) was obtained from Sigma Chemical Co. (St. Louis, M0, USA). A 50 $\mu \mathrm{L}$ aliquot of this mixture was added to $150 \mu \mathrm{L}$ of reaction solution consisting of $100 \mathrm{mM}$ Tris$\mathrm{HCl}$ buffer (pH 7.5), $5 \mathrm{mM}$ dithiothreitol, $5 \mathrm{mM}$ L-cysteine and $1 \mathrm{mM} N$ - $\alpha$-Benzoyl-L-arginine$p$-nitroanilide (BApNA) (Sigma Chemical). The assay mixture was incubated at $37^{\circ} \mathrm{C}$ for $20 \mathrm{~min}$ and the reaction was stopped by adding $50 \mu \mathrm{L}$ of $20 \%$ acetic acid. The release of $\mathrm{p}$ nitroaniline was determined by measuring its absorbance at $405 \mathrm{~nm}$. 16 The reaction without gingipains was used as the negative control to monitor background readings. One unit of gingipain activity was defined as the amount of enzyme releasing $1 \mu \mathrm{mol}$ of $\mathrm{p}$ nitroanilide per minute in the reaction mixture under assay conditions and expressed as $\mathrm{U} / \mathrm{mL}$.

The cell damage assay was based on the protocol of Yokoyama et al. ${ }^{16}$ In this assay, FaDu cells (Human pharyngeal carcinoma cell line; ATCC HTB-43, Manassas, VA, USA) were cultured overnight in minimum essential medium (Eagle's minimum essential medium, Nissui, Japan) containing 10\% FBS in six-well microtiter plates. The plates were charged with serum-free medium containing gingipains (200, 100, 50 and $0 \mu \mathrm{g} / \mathrm{mL})$, phosphate buffered saline (PBS) or gingipains from Porphyromonas spp pretreated with either IgYGP or control IgY (50 mg/mL each) and incubated at $37^{\circ} \mathrm{C}$ for $1 \mathrm{~h}$. The plates were washed 3 times with PBS to remove detached cells and the remaining cells were counted after trypan blue staining. A similar procedure was used on two other cell lines, KB cells (Human pharyngeal carcinoma cell line; ATCC CCL-17, USA.) and $\mathrm{Ca}$ 9-22 cells (Human gingival cell line; Japanese Cell Resource Bank, JCRB-0625, Shinjuku, Japan).

\section{Effect of IgY-GP on dogs}

All procedures that involved animals were approved by the animal care and use committee of animal research center, Kyodoken Institute, Kyoto, Japan. Mixed-breed dogs with different degrees of periodontitis were purchased from the commercial dog breeders. The dogs were housed individually in stainless steel cages in a temperature-controlled room $\left(25^{\circ} \mathrm{C}\right)$ with a 12 -hour light/dark cycle. Food and water were provided ad libitum. The dogs were physically healthy and had not been treated with any antimicrobials prior to the study. The dogs were placed under acclimation period for 7 days, during which they were fed a commercial diet (Aijou monogatari series, Yeaster Co., Hyogo, Japan.), a dry pellet food free of antimicrobials and probiotics.

Two experiments were conducted to examine the effect of IgY-GP on dogs. In the first experiment, 15 dogs were randomly divided into 3 groups (5 dogs per group): two test groups (test 1 and test 2 , respectively) and one control group. The test 1 group (average body weight $=7.88 \pm 1.34 \mathrm{kgs}$ and age $=74 \pm 13$ months) was fed $35 \mathrm{mg}$ IgY-GP per kg of body weight once a day. The test 2 group (average body weight $=8.04 \pm 1.49 \mathrm{kgs}$ and age $=67 \pm 2$ months) was fed $17.5 \mathrm{mg}$ IgY-GP per kg of body weight twice a day. IgY-GP yolk powder was mixed with dry pellet food just prior to feeding and fed to the dogs for 8 weeks. The control group (average body weight $=8.38 \pm 1.91 \mathrm{kgs}$ and age $=68 \pm 2.30$ months) was fed the same dry feed supplemented with control IgY.

One premolar tooth was selected from each dog for examination at baseline (one day before treatment), 4 and 8 weeks post treatment. Neither scaling nor root planning (SRP) was done supra-and subgingivally for these experimental teeth. The examiner was not involved in the treatment process and was not aware of the dog group assignment. The examination parameters included gingivitis and periodontitis scores, bleeding on probe (BOP), pocket depth (PD) and dental calculus removal status. During the oral examination, the dogs were sedated by intramuscular injection of medetomidine hydrochloride $(0.05 \mathrm{mg} / \mathrm{kg})$ (Medetomidine hydrochloride; Domitor; Meiji Seika Kaisha, Tokyo, Japan) and then antisedated with atipamezole hydrochloride $(0.05$ $\mathrm{mg} / \mathrm{kg}$ ) (Atipamezole hydrochloride; Antisedan; Meiji Seika Kaisha). Photographs were taken at the time of oral examination. The gingivitis and periodontitis levels were scored as follow: score 0 = normal; 1 = mild; 2 = moderate; and $3=$ severe inflammation. The oral health index was calculated as the sum of scores with 0 point indicating optimal periodontal health and 6 points or higher indicating poorest periodontal health. ${ }^{18}$ BOP was assessed by the presence or absence of bleeding $30 \mathrm{sec}$ after probing. 14,19 PD was measured by the use of a standard periodontal probe. For evaluation of dental calculus removal status, one premolar tooth per dog was selected. den- tal calculus thickness and areas were assessed through the comparison of photographs taken for representative teeth by three independent veterinarians. Mean values are expressed as dental calculus removal percentages.

In the second experiment, five dogs (average body weight $=6.6 \pm 1.98 \mathrm{kgs}$ and age $=$ $70 \pm 2$ months) were used. Two pairs of contralateral premolar teeth per each dog (4 sites/ dog) were selected to check the effect of IgYGP by a split mouth design.14,19 The selected teeth all had clear gingival inflammation with a PD $\geq 5 \mathrm{~mm}$. One tooth in each contralateral pair was treated with $20 \%$ IgY-GP mixed in dental ointment (a neutral gel containing hydrocarbon gel, sucrose esters of fatty acids and hydroxypropylmethylcellulose 2208) (Showa Yakuhin Kako Co., Ltd., Tokyo, Japan) (test sites, $\mathrm{n}=10$ ), whereas the other tooth was treated with dental ointment only (control sites, $n=10$ ). The dental ointment was administered 4 times ( $200 \mathrm{mg} /$ pocket/time) at weekly interval into the periodontal pocket by a root canal syringe without pretreatment by scaling and root planning (SRP). Clinical parameters were recorded and scored for at baseline, 1, 2, 3 and 4 weeks post treatment as described in the first experiment. PD was measured at baseline, 2 weeks and 4 weeks post treatment.

All data are presented as the means \pm standard deviations (SD). The statistical significance was evaluated by Chi-square, ANOVA and Student's $t$-test where appropriate. A value of $\mathrm{P}<0.05$ was considered to be statistically significant.

\section{Results}

\section{In vitro efficacy of IgY-GP}

The activity of IgY samples was measured by ELISA. The titer of IgY-GP was 128,000 while that of the control IgY was less than 100 . The reactivity of the IgY-GP with different Porphyromonas spp. is shown in the Table 1. IgY-GP strongly reacted with $P$. gingivalis and $P$. gulae but showed weak cross activity with $P$. salivosa. IgY-GP did not cross-react with $P$. cir-

Table 1. Cross-reactivity of IgY-GP with different Porphyromonas species.

\begin{tabular}{lcc}
\hline Porphyromonas species & $\operatorname{IgY}$-GP & Control IgY \\
P. gingivalis ATCC 33277 & $1.276 \pm 0.01^{\circ}$ & $0.015 \pm 0.03$ \\
P. gingivalis 381 & $1.196 \pm 0.01$ & $0.000 \pm 0.02$ \\
\hline P. gulae ATCC 51700 & $0.833 \pm 0.02$ & $0.019 \pm 0.01$ \\
P. salivosa ATCC 49407 & $0.227 \pm 0.04$ & $0.010 \pm 0.01$ \\
\hline P. circumdentaria ATCC 51356 & $0.064 \pm 0.01$ & $0.040 \pm 0.02$ \\
P. endodontalis F2 & $0.094 \pm 0.01$ & $0.010 \pm 0.004$ \\
\hline P. endodontalis F5 & $0.057 \pm 0.02$ & $0.015 \pm 0.002$ \\
\hline
\end{tabular}

Absorbance at $492 \mathrm{~nm}$; ${ }^{\circ}$ the highest dilution of IgY-GP showing an OD of more than 0.2 was used as the cut-off value for a positive reaction;

${ }^{b}$ mean $\mathrm{OD} \pm$ standard deviation. Data shown are the means of three independent experiments. 
cumdentaria and clinical isolates of $P$. endodontalis F2 and F5. Control IgY showed no reaction with all Porphyromonas spp.

Very high proteolytic activity was detected in gingipain preparations isolated from the two $P$. gingivalis strains and $P$. gulae ATCC 51700 strain. Weak proteolytic activity was observed in the preparation from $P$. salivosa ATCC 49407 strain and no such activity was found in the preparations from $P$. circumdentaria ATCC 51356 and the 2 P. endodontalis strains. In the enzyme inhibition assay, IgY-GP inhibited the proteolytic activity of gingipain preparations $(200 \mu \mathrm{g} / \mathrm{mL})$ from P. gulae strain ATCC 51700 and $P$. gingivalis strain 381 by $50 \%$ (Figure 1 ) and $60 \%$, respectively.

In the cell damage assays, gingipains from $P$. gulae added to FADU monolayer cell culture resulted in cell death and subsequent detachment from the plates (Figure 2A) in a dosedependent manner (Figure 2B). Pretreatment of gingipains with IgY-GP protected the cells from damage and significantly increased cell survival compared to the non-treated control groups $(\mathrm{P}<0.05$, one-way ANOVA) whereas that with control IgY did not show any protection effects (Figure 2). Similar results of cell protection were obtained when the 2 other cell lines ( $\mathrm{KB}$ and $\mathrm{Ca} 9-22)$ were used in separate experiments (data not shown).

\section{Effect of IgY-GP on dog}

All the dogs used in the experiments remained healthy throughout the test period. No allergy reaction or any side effects were observed on any dog administered with the test and placebo samples. The effect of IgY-GP on dog oral health status is shown in Table 2 and Table 3 for the first and second experiment, respectively. In the first experiment, the mean scores for all examined parameters including

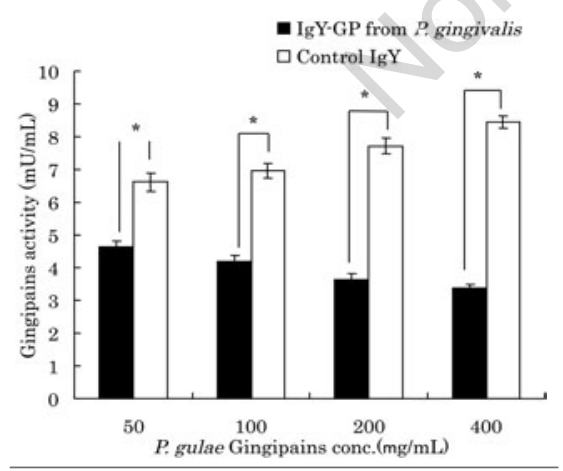

Figure 1. Effect of IgY-GP on the cross protease activities of gingipain extracted from P. gulae. The inhibitory effects of IgY-GP from $P$. gingivalis and control IgY (50 $\mathrm{mg} / \mathrm{mL}$ each) on gingipain activity were evaluated. Data are shown as the means SD of three independent experiments. ${ }^{*} \mathbf{P}<0.01$ indicates a significant difference among study groups according to Student's $t$-test. gingivitis, periodontitis, oral health index, and bleeding on probe, were significantly lower after 8 weeks compared to the baseline $(\mathrm{P}<0.05$ or $\mathrm{P}<0.01$ depending on parameters, one-way ANOVA) (Table 2). There were no significant changes in any parameters in the control group at 4 and 8 weeks. No remarkable differences were observed for all parameters between test 1 and test 2 groups. The effect of IgY-GP on oral health was also clearly seen in experiment 2 where most examined parameters were significantly lower starting from $2^{\text {nd }}$ week post treatment. No significant changes were noticed for any parameter in the control group (Table 3).

Table 2. Effect of IgY-GP supplemented with dry food on oral health parameters of dogs with periodontitis.

\begin{tabular}{lcccccccccc} 
Parameters & \multicolumn{3}{c}{ Test $1^{\circ}(\mathrm{n}=5)$} & \multicolumn{3}{c}{ Test $2 *(\mathrm{n}=5)$} & \multicolumn{2}{c}{ Control $(\mathrm{n}=5)$} \\
& Baseline & $4 \mathrm{wk}$ & $8 \mathrm{wk}$ & Baseline & $4 \mathrm{wk}$ & $8 \mathrm{wk}$ & Baseline & 4 wk & $8 \mathrm{wk}$ \\
Gingivitis $\$$ & $1.2 \pm 0.8$ & $0.7 \pm 0.48$ & $0.2 \pm 0.42^{*}$ & $1.2 \pm 0.42$ & $0.7 \pm 0.48^{* *}$ & $0.8 \pm 0.42^{* *}$ & $1.7 \pm 0.95$ & $1.6 \pm 0.84$ & $1.5 \pm 0.97$ \\
Periodontitis $^{\wedge}$ & $1.1 \pm 1.1$ & $0.1 \pm 0.32 * 0.0 \pm 0.0^{*}$ & $1.2 \pm 1$ & $0.2 \pm 0.42^{*}$ & $0.2 \pm 0.42^{*}$ & $1.4 \pm 1.1$ & $0.9 \pm 1.2$ & $0.8 \pm 1.0$ \\
\hline Oral health index $^{\$}$ & $2.3 \pm 0.07$ & $0.8 \pm 0.4$ & $0.2 \pm 0.1$ & $2.4 \pm 0$ & $0.9 \pm 0.35$ & $1.0 \pm 0.42$ & $3.1 \pm 0.2$ & $2.5 \pm 0.5$ & $2.3 \pm 0.5$ \\
Bleeding on probe $^{\circ}$ & $4 / 5$ & $1 / 5$ & $1 / 5$ & $5 / 5$ & $0 / 5$ & $0 / 5^{*}$ & $5 / 5$ & $5 / 5$ & $5 / 5$
\end{tabular}

${ }^{\circ}$ The test 1 group was fed $35 \mathrm{mg}$ IgY-GP per kg of body weight once a day; "the test 2 group was fed $17.5 \mathrm{mg}$ IgY-GP per kg of body weight twice a day; $\$ \wedge, \$$,gingivitis and periodontitis levels were evaluated visually and scored as follow: score $0=$ normal; $1=$ mild; $2=$ moderate and $3=$ severe inflammation. The oral health index was calculated as the sum of gingivitis and periodontitis scores with 0 point indicating optimal periodontal health and 6 points or higher indicating poorest periodontal health. ${ }^{\circ}$ Bleeding on probe was assessed by the presence or absence 0 bleeding $30 \mathrm{sec}$ after probing. Values reported are no. of sites with bleeding/no. of sites examined. ${ }^{*} \mathrm{P}<0.01,{ }^{* *} \mathrm{P}<0.05$ compared to baseline.

A)
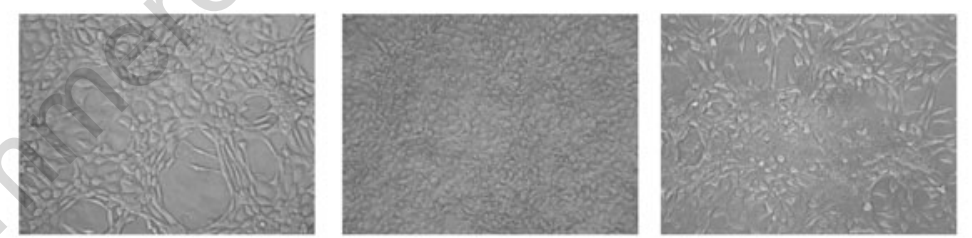

Gingipain only

Gingipain pretretaed with IgY-GP Gingipain pretretaed with control IgY

B)

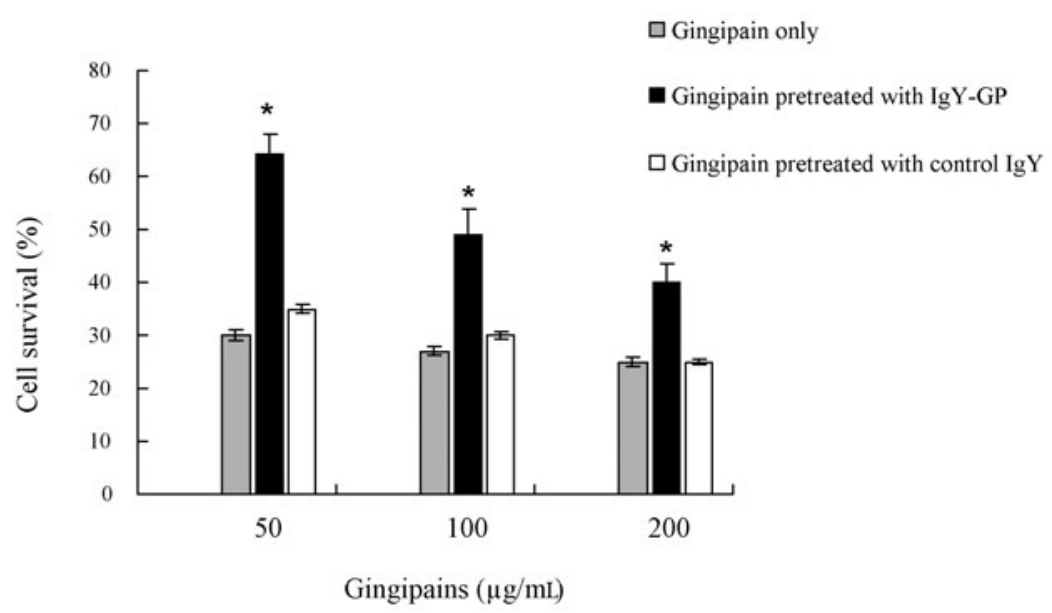

Figure 2. Effect of IgY-GP on the epithelial cell attachement. A) Microphotographs of the morphological changes of $\mathrm{FaDu}$ cells treated with gingipains extracted from P. gulae and pretreated with IgY-GP or control IgY ( $\times 200$ magnification). B) Inhibition of gingipaininduced cell damage by IgY-GP or control IgY. Data are shown as the means \pm SD of three independent experiments. ${ }^{*} \mathrm{P}<0.01$ (Student's $t$-test) indicates significant differences between the IgY-GP compared to the control IgY groups. 
( $\mathrm{P}<0.05$, Student's $\mathrm{t}$ test). The dental calculus removal pattern results in the first experiment are shown in the Table 4 . At 4 weeks post treatment, 3 and 1 dog in the test 1 and test 2 groups, respectively, demonstrated dental calculus removal with the reduction of tooth surface areas of $26 \%$ and $4 \%$, respectively. The figures for the 2 groups at 8 weeks were 4 and 2 dogs, respectively, and $42 \%$ and $22 \%$ reduction in dental calculus area, respectively. No dental calculus removal was seen in the control group. There was a significant difference in the number of dogs with dental calculus removal between the test 1 and control group at 8 weeks post treatment $\left(\mathrm{P}<0.05, \chi^{2}\right)$. Figure 4 shows the change in dental calculus removal pattern in 3 dogs in the test groups. At 8 weeks these dogs show clear reduction of dental calculus build-up on the teeth.

\section{Discussion}

This study was undertaken to investigate the in vitro and in vivo efficacy of egg yolk antibody against $P$. gingivalis gingipains (IgYGP) in prevention and treatment of gum diseases in dogs. The most significant finding in this study is the fact that IgY-GP in various experiment settings resulted in significant improvement of oral health status in the dogs.

Black-pigmented bacteria have been known to cause periodontitis and gum diseases in human and animals. Several bacterial species have been reported to be the causative agents of periodontitis in dogs which include $P$. ging $i$ valis, $P$. gulae, and others. ${ }^{20}$ Although gingipain is known to be an important virulent factor of these bacteria, no studies to compare the enzyme from different black-pigmented bacteria have been conducted to date. In the present study we demonstrated for the first time that IgY against gingipain from $P$. gingivalis crossreacts with the same enzyme expressed by $P$. gulae and protects various cell lines from damages by the enzymes expressed by both $P$. gingivalis and $P$. gulae. The IgY did not react with $P$. circumdentaria and $P$. endodontalis isolates because these bacteria are non-protease bearers. The role of these bacteria in periodontitis development is uncertain and need to be investigated.

To investigate the efficacy of IgY-GP in dogs we designed 2 sets of experiments. In the first experiment we checked the preventive effect of IgY-GP on the dogs by mixing the IgY samples directly with dry pellet food. We choose 2 different feeding regimes based on an assumption that $\operatorname{IgY}$ concentration and retention in the mouth cavity could potentially affect the outcome. In both feeding regimes IgY-GP showed comparable effect on oral health status which is demonstrated by significant improve- ment of important parameters such as gingivitis, periodontitis, BOP, and oral health index. These results indicate that IgY-GP is useful in reduction of inflammation in oral cavity and prevention of periodontitis and gum diseases in dogs. The effect of IgY-GP on dental calculus removal pattern was exciting because dental calculus on teeth can be removed only by mechanical methods. Although the mechanism behind this effect is still unclear, specific IgY against gingipain may weaken the biofilm formed on teeth surface and result in the change in structure of dental calculus. The better dental calculus removal pattern in the test 1 compared to the test 2 group suggests that higher concentration of IgY-GP in oral cavity may be necessary to prevent tartar build up on teeth surface.

Although feeding IgY-GP with dry feed resulted in reduction of inflammation in dog oral cavity, the antibody did not have an effect on PD after 8 weeks of continuous feeding
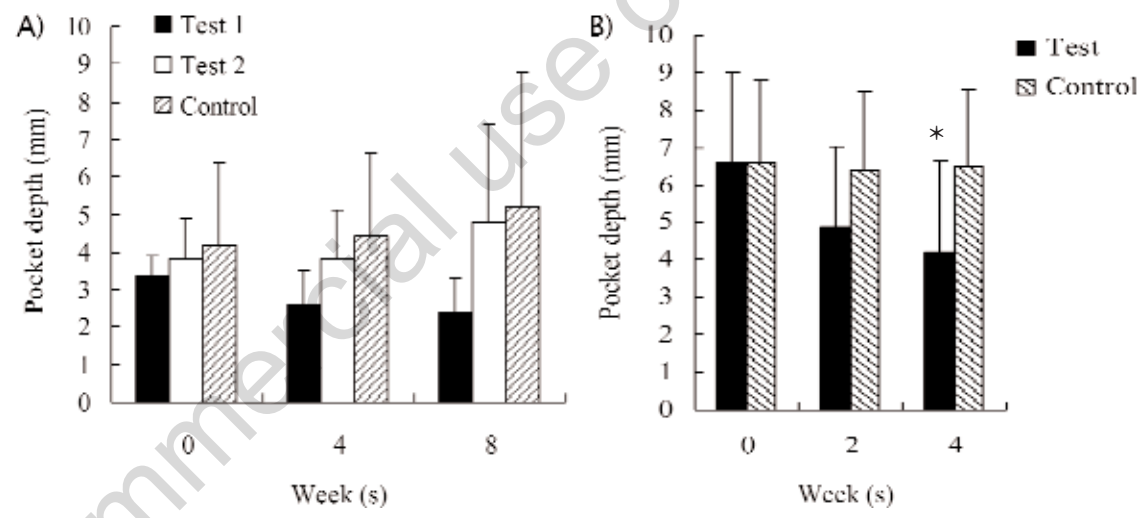

Figure 3. Effect of IgY-GP on the changes in probing depth in dogs when supplemented with A) dry food in experiment 1 and with B) dental ointment in experiment 2 . Results are means $\pm S D ;{ }^{*} \mathbf{P}<0.05$ indicates a significant difference between the test and control groups.

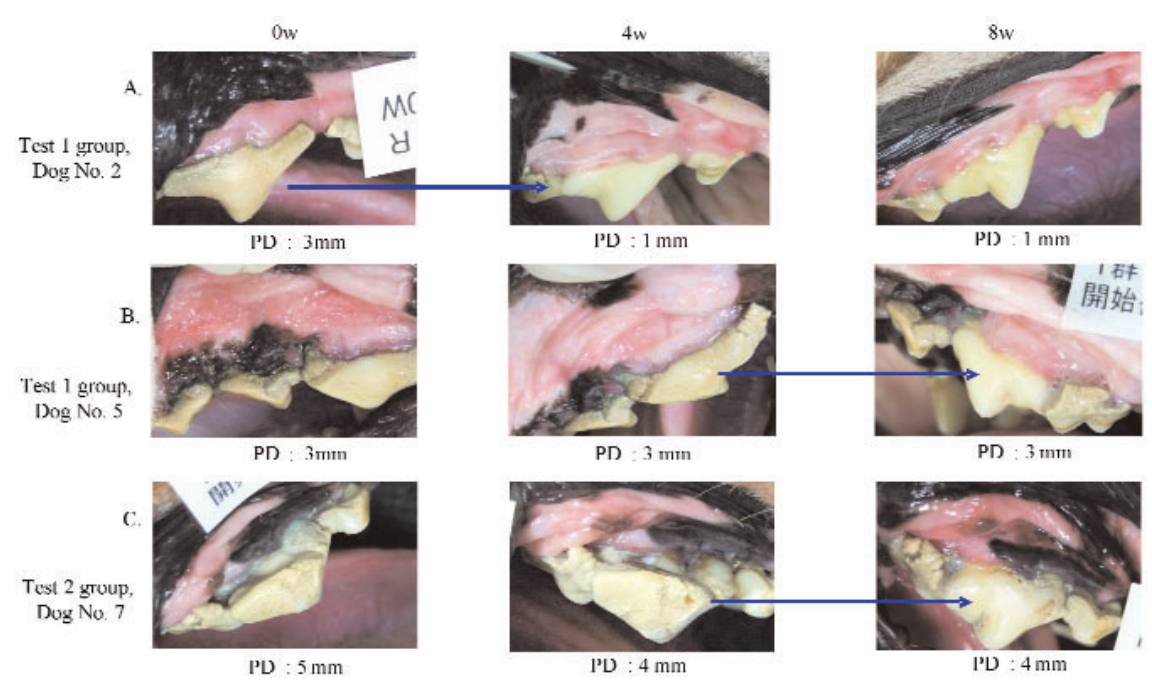

Figure 4. Representative dental photographs of 3 experimental dogs (A, B and C) showing the effect of IgY-GP on dental calculus removal. The same tooth was photographed before, 4 weeks and 8 weeks after feeding with the IgY-GP. PD, pocket depth. 
Table 3. Effect of IgY-GP supplemented with dental ointment on oral health parameters of dogs with periodontitis.

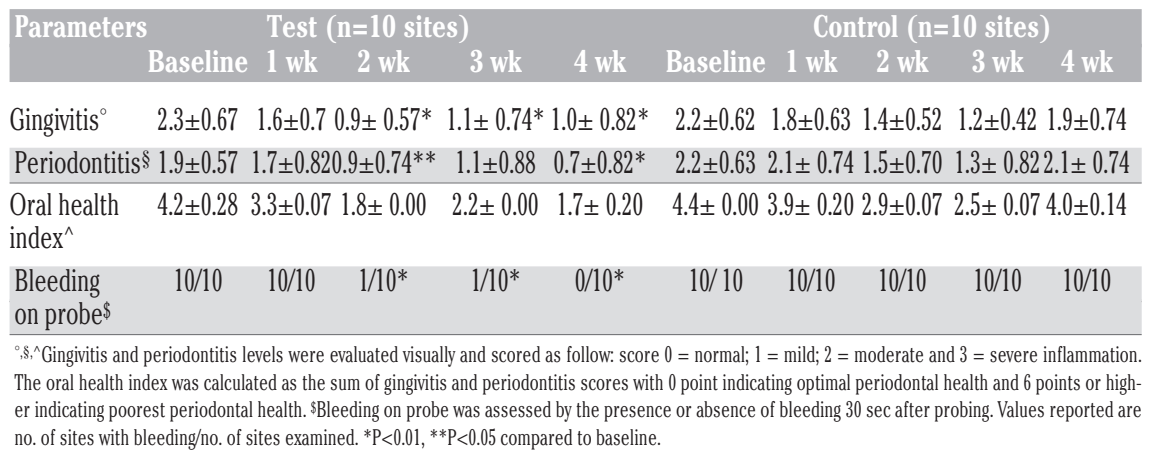

Table 4. Dental calculus removal pattern in dogs fed with IgY-GP (Test 1 and 2) or control IgY-supplemented feed.

\begin{tabular}{|c|c|c|}
\hline Group & $\begin{array}{l}\text { jental c } \\
4 \text { weeks }\end{array}$ & $\begin{array}{l}\text { Jattern (\%) on tooth surfaces } \\
8 \text { weeks }\end{array}$ \\
\hline Test $1^{\circ}(\mathrm{n}=5)$ & $3(26 \%)$ & $4^{*}(42 \%)$ \\
\hline Test $2^{\#}(n=5)$ & $1(4 \%)$ & $2(22 \%)$ \\
\hline Control & $0(0 \%)$ & $0(0 \%)$ \\
\hline
\end{tabular}

this is the first report on the use of IgY-GP for prevention and treatment of periodontitis in dogs. Compared to antimicrobial drugs IgY from chickens has various advantages due to its specific activity and its localized and noninvasive nature, which make IgY very safe especially when used for a long period of time. ${ }^{13,22}$ In our study we also did not see any side effects in dogs during 8 weeks of continuous administration of the IgY. This fact together with the effects on various inflammationrelated parameters indicates that IgY-GP is a valuable tool for prevention and adjunctive treatment of periodontitis in dogs.

\section{Conclusion}

It is concluded that oral immunotherapy by using IgY-GP is a new promising alternative to conventional preventive and therapeutic methods to improve oral health status in dogs.

\section{References}

1. Gorrel C. Periodontal disease and diet in domestic Pets. J Nutr 1998;128:S2712-14.

2. Socransky SS, Haffajee AD, Cugini MA, Smith C, Kent RL Jr. Microbial complexes in subgingival plaque. J Clin Periodontol 1998;25:134-44.

3. Baker PJ, Evans RT, Roopenian DC. Oral infection with Porphyromonas gingivalis and induced alveolar bone loss in immuno- competent and severe combined immunodeficient mice. Arch Oral Biol 1994;39: 1035-40.

4. Katz J, Ward DC, Michalek SM. Effect of host responses on the pathogenicity of strains of Porphyromonas gingivalis. Oral Microbiol Immunol 1996;5:309-18.

5. Persson GR, Engel D, Whitney G, et al. Immunization against Porphyromonas gingivalis inhibits progression of experimental periodontitis in nonhuman primates. Infect Immun 1994;62:1026-31.

6. Genco CA, Potemp J, MikolajczykPawlinska J, Travis J. Role of gingipains $\mathbf{R}$ in the pathogenesis of Porphyromonas gingivalis-mediated periodontal disease. Clin Infect Dis 1999;28:456-65.

7. Rajapakse PS, O'Brien-Simpson NM, Slakeski N, et al. Immunization with the Rgp-Kgp proteinase-adhesin complexes of Porphyromonas gingivalis protects against periodontal bone loss in the rat periodontitis model. Infect Immun 2002;70:2480-86.

8. Pike R, McGraw W, Potempa J, Travis J. Lysine- and arginine-specific proteinases from Porphyromonas gingivalis: isolation, characterization, and evidence for the existence of complexes with hemagglutinins. J Biol Chem 1994;269:406-11.

9. Ryosuke T, Tomoko K, Atsuyo B, et al. A Functional virulence complex composed of gingipains, adhesins, and lipopolysaccharide shows high affinity to host cells and matrix proteins and escapes recognition by host immune systems. Infect Immun 2005;73:883-93.

10. Larsson A, Carlander D. Oral immunother- apy with yolk antibodies to prevent infections in humans and animals. Ups $\mathrm{J}$ Med Sci 2003;108:129-40.

11. Sa NV, Umeda K, Yokoyama H, Tohya Y, Kodama Y. Passive protection of dogs against clinical disease due to canine parvovirus- 2 by specific antibody from chicken egg yolk. Can J Vet Res 2006;70:62-4.

12. Suzuki H, Nomura S, Masaoka T, et al. Effect of dietary anti-Helicobacter pyloriurease immunoglobulin Y on Helicobacter pylori infection. Aliment Pharmacol Ther 2004;20:185-92.

13. Carlander D, Kollberg H, Wejaker PE, Larsson A. Peroral immunotherapy with yolk antibodies for the prevention and treatment of enteric infections. Immunol Res 2000;21:1-6.

14. Yokoyama K, Sugano N, Shimada T, et al. Effects of egg yolk antibody against Porphyromonas gingivalis gingipains in periodontitis patients. J Oral Sci 2007;49:201-6.

15. Isogai H, Kosako Y, Benno Y, Isogai E. Ecology of genus Porphyromonas in canine periodontal disease. J Vet Med B 1999;46: 467-73.

16. Yokoyama K, Sugano N, Rahman AK, et al. Activity of anti-Porphyromonas gingivalis egg yolk antibody against gingipains in vitro. Oral Microbiol Immunol 2007;22:3525.

17. Kuroki M, Ikemori Y, Yokoyama H, et al. Passive protection against bovine rotavirusinduced diarrhea in murine model by specific immunoglobulins from chicken egg yolk. Vet Microbiol 1993;37: 135-46.

18. Gawor JP, Reiter AM, Jodkowska K, et al. Influence of diet on oral health in cats and dogs. J Nutr 2006;136:S2021-23S.

19. de Oliveira RR, Schwartz-Filho HO, Novaes ABJr, Taba MJr. Antimicrobial photodynamic therapy in the non-surgical treatment of aggressive periodontitis: A preliminary randomized controlled clinical study. J Periodont 2007;78:965-73.

20. Hardham J, Dreier K, Wong J, et al. Pigmented-anaerobic bacteria associated with canine periodontitis. Vet Microbiol 2005;106:119-28.

21. Hamada N, Watanabe K. The egg yolk antibody against gingipains protects Porphyromonas gingivalis-induced boneless in rats. Proc. of 96th Ann. Meet. Am. Acad. Periodontol 2010, poster no. 86579.

22. Nilsson E, Kollberg H, Johannesson M, et al. More than 10 years' continuous oral treatment with specific immunoglobulin $\mathrm{Y}$ for the prevention of Pseudomonas aeruginosa infections: a case report. J Med Food 2007;10:375-8. 